\title{
Time-resolved metabolomics reveals metabolic modulation in rice
} foliage

Shigeru Sato ${ }^{1}$, Masanori Arita ${ }^{1,2,3}$, Tomoyoshi Soga ${ }^{1,4}$, Takaaki Nishioka ${ }^{1,5}$ and Masaru Tomita*1,4

Address: ${ }^{1}$ Institute for Advanced Biosciences, Keio University, Tsuruoka, Japan, ${ }^{2}$ Department of Computational Biology, Graduate School of Frontier Sciences, The University of Tokyo and PRESTO-JST, Kashiwa, Japan, ${ }^{3}$ Plant Science Center, Riken, Yokohama, Japan, ${ }^{4}$ Human Metabolome Technologies, Inc., Tsuruoka, Japan and ${ }^{5}$ Graduate School of Agriculture, Kyoto University, Kyoto, Japan

Email: Shigeru Sato -n03615ss@sfc.keio.ac.jp; Masanori Arita - arita@k.u-tokyo.ac.jp; Tomoyoshi Soga - soga@sfc.keio.ac.jp; Takaaki Nishioka - takaaki@sfc.keio.ac.jp; Masaru Tomita* - mt@sfc.keio.ac.jp

* Corresponding author

Published: 18 June 2008

BMC Systems Biology 2008, 2:5 I doi:10.1 |86/1752-0509-2-5 I
Received: 12 February 2008

Accepted: 18 June 2008

This article is available from: http://www.biomedcentral.com/1752-0509/2/5I

(C) 2008 Sato et al; licensee BioMed Central Ltd.

This is an Open Access article distributed under the terms of the Creative Commons Attribution License (http://creativecommons.org/licenses/by/2.0), which permits unrestricted use, distribution, and reproduction in any medium, provided the original work is properly cited.

\begin{abstract}
Background: To elucidate the interaction of dynamics among modules that constitute biological systems, comprehensive datasets obtained from "omics" technologies have been used. In recent plant metabolomics approaches, the reconstruction of metabolic correlation networks has been attempted using statistical techniques. However, the results were unsatisfactory and effective datamining techniques that apply appropriate comprehensive datasets are needed.
\end{abstract}

Results: Using capillary electrophoresis mass spectrometry (CE-MS) and capillary electrophoresis diode-array detection (CE-DAD), we analyzed the dynamic changes in the level of 56 basic metabolites in plant foliage (Oryza sativa L. ssp. japonica) at hourly intervals over a 24-hr period. Unsupervised clustering of comprehensive metabolic profiles using Kohonen's self-organizing map (SOM) allowed classification of the biochemical pathways activated by the light and dark cycle. The carbon and nitrogen $(\mathrm{C} / \mathrm{N})$ metabolism in both periods was also visualized as a phenotypic linkage map that connects network modules on the basis of traditional metabolic pathways rather than pairwise correlations among metabolites. The regulatory networks of $\mathrm{C} / \mathrm{N}$ assimilation/ dissimilation at each time point were consistent with previous works on plant metabolism. In response to environmental stress, glutathione and spermidine fluctuated synchronously with their regulatory targets. Adenine nucleosides and nicotinamide coenzymes were regulated by phosphorylation and dephosphorylation. We also demonstrated that SOM analysis was applicable to the estimation of unidentifiable metabolites in metabolome analysis. Hierarchical clustering of a correlation coefficient matrix could help identify the bottleneck enzymes that regulate metabolic networks.

Conclusion: Our results showed that our SOM analysis with appropriate metabolic time-courses effectively revealed the synchronous dynamics among metabolic modules and elucidated the underlying biochemical functions. The application of discrimination of unidentified metabolites and the identification of bottleneck enzymatic steps even to non-targeted comprehensive analysis promise to facilitate an understanding of large-scale interactions among components in biological systems. 


\section{Background}

In the post-genome era, comprehensive data from "omics" technologies (genomics, transcriptomics, proteomics, and metabolomics) have been extensively analyzed to elucidate the underlying biochemical networks that elaborately regulate cellular mechanisms. Recent contributions from metabolomics are particularly noteworthy; they offer insights into metabolism that complement information obtained from proteomics and transcriptomics [1]. Correlation analysis of metabolic profiles has been used effectively to distinguish silent phenotypes or genetic alterations that are not noticeable superficially [2-4]. The systematic integration of metabolomic-, proteomic-, and transcriptomic profiles facilitates the unbiased, information-based reconstruction of underlying biochemical networks [5,6]. Kohonen's self-organizing map (SOM) analysis [7] was also an effective method to classify and monitor metabolic alteration patterns with time-series profiles [8,9].

However, with the current technology, unbiased reconstruction from comprehensive and high-throughput data is challenging; statistical tools are immature and inherent measurement errors and biological noise continue to present problems [10]. Moreover, two issues are relevant to the exploitation of metabolomics data. First, it is crucial to interpret metabolic profiles by focusing on a specific rhythm in an appropriate time range and interval, since plants have adapted their metabolism to different environmental fluctuations such as the slow and steady diurnal rhythm, whereas metabolic levels change dynamically. Second, currently available metabolomics data are insufficient for the detection of new metabolic networks. Even if non-target profiling were able to quantify thousands of metabolites, at present there is no method for estimating their reliability. As statistical inference requires large amounts of data measured under similar conditions in transcriptomics [11], the verification of network dynamics for known pathways must precede attempts to identify unknown network structures. It appears that each metabolic profile is measured under method-specific, presumably biased conditions.

Time-resolved target analysis is an effective way to observe biochemical dynamics. We systematically measured the level of 56 basic metabolites in rice leaves (Oryza sativa L. ssp. japonica) at hourly intervals over a 24 -hr period. Our target and experimental conditions were strategically determined: 1) we focused on primary metabolic pathways consisting of carbon fixation/respiration- and nitrogen assimilation/dissimilation pathways, and comprehensively quantified related metabolites, 2) the photocycle was the sole environmental factor, and 3) measurements were made at 1-hr intervals to allow the observation of dynamic profiles.
High-throughput analysis was conducted with the capillary electrophoresis - mass spectrometry (CE-MS) technology we developed earlier [12-14], and has been applied to metabolic profiling in Bacillus subtilis extracts [15] and monitoring of genetic and environmental perturbations in Escherichia coli cells [16]. Each employed CEMS method was able to detect charged low molecular metabolites in less than $30 \mathrm{~min}$ without requiring derivatization. Combined with diode array detection (CEDAD), our technology is also applicable to quantifying small sugar compounds. We previously developed a sample preparation protocol that could extract metabolites with possibly minimal metabolic turnover [17]. By using the CE-MS and CE-DAD, we also succeeded in analyzing over eighty major metabolites (sugars, organic acids, amino acids, and nucleotides) in rice foliage. The current work is our first systematic time-course measurements of rice foliage throughout a day.

We applied four information-based methods to analyze the diurnal fluctuation of metabolites: 1) metabolic pathways were classified with SOM to monitor the metabolic dynamics in each time-step, 2) a phenotypic linkage map was constructed from the classified pathways by Sammon's 2D-network layout [18], 3) unidentified metabolites were predicted based on SOM analysis and chemical structures, and 4) rate-limiting enzymes were identified by hierarchical clustering on a correlation matrix. Here we show that combining metabolome analysis and information-based methods is an effective way to elucidate phenotypical metabolic network structures and underlying biological functions under diurnal rhythm fluctuations.

\section{Results}

\section{Time-course data acquisition}

We extracted target metabolites existing in the primary metabolism such as the glycolytic pathway, the reductiveand oxidative pentose phosphate pathway, and the photorespiratory pathway, the tricarboxylic acid (TCA) cycle, and the amino acid biosynthetic pathway. Figure 1 presents the practical rice biochemical network that was constructed with our target metabolites based on annotated protein data from the KEGG pathway database [19], Swiss-Prot database [20], or Rice Annotation Project Data Base [21]. It shows the names of target metabolites and the EC number of enzymatic reactions; black dots are non-target metabolites. Although $\mathrm{NH}_{3}$ (also $\mathrm{R}-\mathrm{NH}_{2}$ ) and $\mathrm{CO}_{2}$ were non-target compounds, they are shown in green to demonstrate in and out of carbon and nitrogen.

We selected eight enzymatic proteins that have not been annotated at this stage to determine whether they function in the rice plant. These enzymes and the judgment criteria are shown in Table 1 . On the map, their EC numbers and lines are presented in gray. 


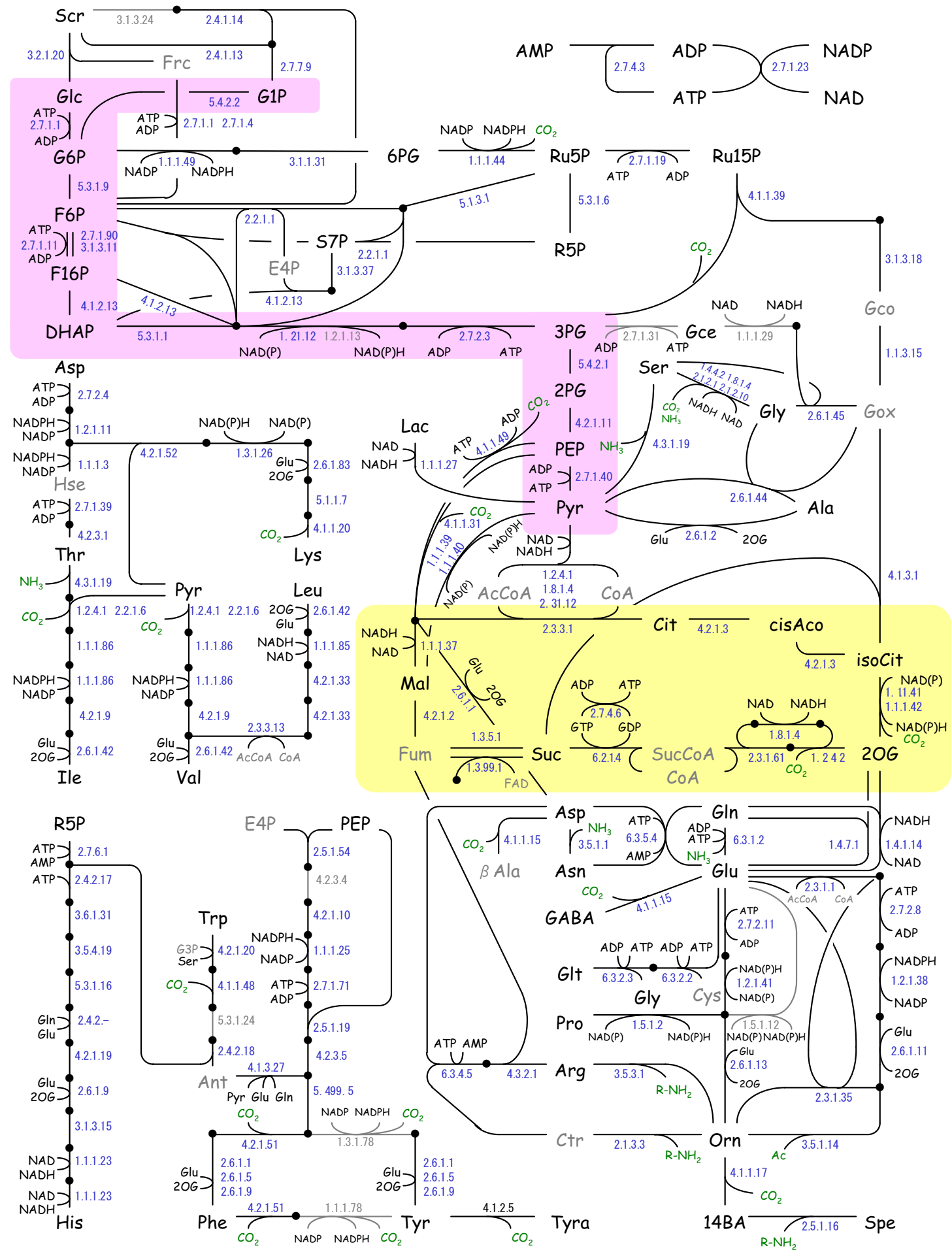

Figure I

Metabolic network of oryza sativa L. ssp. Japonica. Target metabolites and practical enzymatic reactions are shown. The number next to the line is the EC number. Colors indicate the ratio of metabolic levels in light and dark periods. Unidentified metabolites are gray and gray lines and EC numbers identify non-annotated enzymatic proteins. The red-and yellow shade show the glycolytic pathway and the TCA cycle respectively. 
Table I: Selected non-annotated proteins expected to function in rice plant

\begin{tabular}{|c|c|c|c|}
\hline EC Number & Enzyme name & Criterion for judgement & Ref. \\
\hline \multirow[t]{2}{*}{ I.I.1.29 } & $\begin{array}{l}\text { hydroxypyruvate reductase; glycerate } \\
\text { dehydrogenase }\end{array}$ & $\begin{array}{l}\text { Enzymatic reduction of hydroxypyruvic acid to D-glyceric acid in } \\
\text { higher plants, i.e. the leaves of pea, beet, tomato, radish, spinach, } \\
\text { parsley, lettuce, corn, kohlrabi, and carrot. }\end{array}$ & [22] \\
\hline & & AK069655; Similar to 2-hydroxyacid dehydrogenase & RAP-DB*I \\
\hline 1.2.1.13 & $\begin{array}{l}\text { glyceraldehyde-3-phosphate } \\
\text { dehydrogenase }\end{array}$ & $\begin{array}{l}\text { AK07I 685; Similar to GADPH (383AA) (Fragment). AK } 67755 \text {; } \\
\text { Similar to Glyceraldehyde-3-phosphate dehydrogenase (EC } \\
\text { I.2.I.I3) (Fragment). }\end{array}$ & RAP-DB \\
\hline \multirow[t]{2}{*}{ I.3.1.78 } & $\begin{array}{l}\text { arogenate dehydrogenase; prehenate } \\
\text { dehydrogenase }\end{array}$ & $\begin{array}{l}\text { TyrAATI (AF43468I) and TyrAAT2(AF434682) in Arabidopsis } \\
\text { thaliana catalyze the oxidative decarboxylation of arogenate into } \\
\text { Tyr in the presence of NADP. TyrAAT also exhibits prephenate } \\
\text { dehydrogenase activity. }\end{array}$ & [23] \\
\hline & & $\begin{array}{l}\text { Q5Z9H5_ORYSJ; Q5Z9H3_ORYSJ; Q5Z6YI_ORYSJ, Putative } \\
\text { arogenate dehydrogenase isoform } 2\end{array}$ & Swiss-Prot/TrEMBL*2 \\
\hline 1.5.1.12 & $\begin{array}{l}\text { delta-I-pyrroline-5-carboxylate } \\
\text { dehydrogenase }\end{array}$ & $\begin{array}{l}\text { AKI21765; Similar to delta-I-pyrroline-5-carbozylate } \\
\text { dehydrogenase }\end{array}$ & RAP-DB \\
\hline 2.7.I.3I & D-glycerate 3-kinase & $\begin{array}{l}\text { GLYK family protein was purified and sequenced from Arabidopsis } \\
\text { thaliana, identified as putative kinase-annotated single-copy gene } \\
\text { At I g8038. This article suggests that an Olyza sativa PRK/UK-like } \\
\text { protein, BAD73764, Os0 I g48990 is grouped with the GLYK kinase } \\
\text { family. }\end{array}$ & [24] \\
\hline 3.1.3.24 & sucrose-phosphatase & AK063330, AK07I525, AK064563; Similar to sucrose-phosphatase & RAP-DB \\
\hline \multirow[t]{2}{*}{ 4.2.3.4 } & 3-dehydroquinate synthase & $\begin{array}{l}\text { Pentafunctional aroma enzyme in Saccharomyces cervisiae includes } \\
\text { EC 4.2.3.4, EC 4.2.1.10, EC 2.5.I.19, EC I.I.I.25, and EC 2.7.I.7I. }\end{array}$ & {$[25]$} \\
\hline & & $\begin{array}{l}\text { AK071977; Similar to 3-dehydroquinate synthase-like protein (EC } \\
\text { 4.2.3.4). Four other proteins were annotated. }\end{array}$ & RAP-DB \\
\hline 5.3.1.24 & phosphoribosyl-anthranilate isomerase & J075072K08; Similar to phosphoribosylanthranilate isomerase & RAP-DB \\
\hline
\end{tabular}

*I: Rice Annotation Project Data Base [2I]

*2: UniProt Knowledge base: Swiss-Plot and TrEMBL [20]

Sedoheptulose 1,7-bisphosphate (S17P) in the pentose phosphate pathway was not identified because the standard reagent was unavailable. Xylulose 5-phosphate (X5P) is a stereoisomer of Ribulose 5-phosphate (Ru5P) and their peak overlap in CE-MS analysis makes the identification even more difficult. Glyceraldehyde 3-phosphate (G3P) and oxaloacetate (OAA) were not accurately determined too, because they were readily reacted or decomposed.

The seventy selected target metabolites were classified into four groups according to their chemical structure-based physiochemical characteristics (Table 2). Group A contained amino acids and amines, group B organic acids and sugar phosphates, group $\mathrm{C}$ nucleotides and coenzymes, and group D sugars. Groups A, B, and C, consisting of ionic substances, were analyzed with three CE-MS methods for cationic, anionic, and nucleotide metabolites; analysis of group D was with a CE-DAD method. For CE separation, we used conventional sample preparation with simple and universal procedures without any derivatization process. As common preparation procedures were applicable under the four analytical conditions, we were able to determine simultaneously a wide variety of chemical compounds.
Plant seedlings were grown under a 13-hr light - 11-hr dark photocycle for 20 to 21 days. The level of the 56 metabolites was successfully quantified at hourly intervals over the course of $24 \mathrm{hr}$. We could identify the peak and determine the peak area for S7P but could not quantify its level, since the reagent was not available at the time of our CE-MS measurement; we later qualitatively identified its peak with the migration time ratio $\left(\mathrm{MT} / \mathrm{MT}_{\mathrm{IS}}\right)$ of S7P to PIPES (internal standard). The other 13 metabolites were under the detection limit (signal-to-noise ratio $(\mathrm{S} / \mathrm{N})<3$ ); their names were colored gray in Figure 1.

In the course of $24 \mathrm{hr}$, the metabolites exhibited various fluctuations (Figure 2). Ru15P, the precursor of carbon fixation, manifested a variation synchronous with the photoperiod; its intracellular concentration increased under illumination and decreased in darkness. Several metabolites exhibited similar light-dependent variations in the reductive pentose phosphate pathway (3PG, R5P, and Ru5P), the glycolytic pathway (3PG, 2PG, PEP, Pyr), the TCA cycle (2OG, Suc, and Mal), and in sugars (Scr and Glc). Citrate, on the other hand, manifested opposite fluctuation changes. In the amino acid biosynthesis pathway, major amino acids (Ala, Asn, Gln, Glu, Gly, and Ser) accumulated during the light period. Minor amino acids that 
Table 2: The 70 target metabolites subjected to analysis of time-resolved dynamics and their abbreviation used in this article

\begin{tabular}{|c|c|c|c|c|c|}
\hline \multicolumn{2}{|c|}{ Group A (CE-MS No.I) } & \multicolumn{2}{|c|}{ Group B (CE-MS No.2) } & \multicolumn{2}{|c|}{ Group C (CE-MS No.3) } \\
\hline \multicolumn{2}{|c|}{ Amino acids } & \multicolumn{2}{|c|}{ Organic acids } & \multicolumn{2}{|c|}{ Nucleotides } \\
\hline Ala & Alanine & cisAco & cis-Aconitate & AMP & AMP \\
\hline$\beta \mathrm{Ala}$ & $\beta$-Alanine & Cit & Citrate & ADP & ADP \\
\hline GABA & $\gamma$-Aminobutyrate & isoCit & iso-Citrate & ATP & ATP \\
\hline Ant & Anthranilate & DHAP & Dihydroxyacetonephosphate & GDP & GDP \\
\hline Arg & Arginine & Fum & Fumarate & GTP & GTP \\
\hline Asn & Asparagine & Gce & Glycerate & \multicolumn{2}{|c|}{ Coenzymes } \\
\hline Asp & Aspartate & Gco & Glycolate & NAD & NAD \\
\hline Ctr & Citrulline & Gox & Glyoxylate & $\mathrm{NADH}$ & $\mathrm{NADH}$ \\
\hline Cys & Cysteine & Lac & Lactate & NADP & NADP \\
\hline Glu & Glutamate & Mal & Malate & NADPH & NADPH \\
\hline Gln & Glutamine & $2 O G$ & 2-Oxoglutarate & CoA & CoA \\
\hline Glt & Glutathione red. & PEP & Phosphoenolpyruvate & $\mathrm{AcCoA}$ & Acetyl-CoA \\
\hline Gly & Glycine & 6PG & 6-Phosphogluconate & SucCoA & Succinyl-CoA \\
\hline $\mathrm{His}$ & Histidine & 2PG & 2-Phosphoglycerate & & \\
\hline Hse & Homoserine & 3PG & 3-Phosphoglycerate & & \\
\hline Leu & Leucine & Pyr & Pyruvate & \multicolumn{2}{|c|}{ Group D (CE-DAD) } \\
\hline Ile & iso-Leucine & Suc & Succinate & \multicolumn{2}{|l|}{ Sugars } \\
\hline Lys & Lysine & \multicolumn{2}{|c|}{ Sugar Phosphate } & Frc & Fructose \\
\hline Orn & Ornithine & E4P & Erythrose 4-phosphate & Glu & Glucose \\
\hline Phe & Phenylalanine & $\mathrm{FI} 6 \mathrm{P}$ & Fructose I,6-bisphosphate & Suc & Sucrose \\
\hline Pro & Proline & F6P & Fructose 6-phosphate & & \\
\hline Ser & Serine & GIP & Glucose I-phosphate & & \\
\hline Thr & Threonine & G6P & Glucose 6-phosphate & & \\
\hline $\operatorname{Trp}$ & Tryptophan & R5P & Ribose 5-phosphate & & \\
\hline Tyr & Tyrosine & RuI5P & Ribulose I,5-bisphosphate & & \\
\hline Val & Valine & Ru5P & Ribulose 5-phosphate & & \\
\hline Amines & & S7P & Sedoheptulose 7-ohosphate & & \\
\hline I4BA & I,4-Butanediamine & & & & \\
\hline Spe & Spermidine & & & & \\
\hline Tyra & Tyramine & & & & \\
\hline
\end{tabular}

are synthesized from specific organic acids through several reaction steps (His, Ile, Leu, Lys, Phe, Trp and Val) accumulated during the dark period.

Table 3 shows the status of adenine nucleosides and nicotinamide coenzymes in the light and dark periods. Whereas the ratios of ADP, NADP, and NADH were almost equal in the light and dark periods, the ratios of AMP and NADPH were higher and those of ATP and NAD were lower in the light period (see Discussion).

\section{Self-organizing map and phenotypic linkage of metabolic modules}

To visualize the functioning networks throughout a 24-hr period, we classified the metabolites according to similarities in their time-dependent behavior by using Kohonen's self-organizing map (SOM) and Sammon's 2D-network layout (Sammon map). The time-dependent levels of each metabolite were represented as a 24-dimensional vector. On the SOM, the 57 metabolites were classified into a 24 $\times 24$ lattice on the basis of vector similarity. The map was roughly divided into two major groups (see the dark gray line in Figure 3A). Metabolites with high levels in the light period are in the left area; those with high levels in the dark period are on the right in the map. On the SOM, each group was further classified and assigned to subgroups consisting of nitrogen- and carbon-assimilating compounds. Certain amino acids were arranged near their precursor organic acids, e.g., Glu/2OG. Gly, Ser, and Ala were grouped with synthetic pathway intermediates such as Pyr and Gce. The degree of similarity among metabolites was quantitatively visualized on the Sammon map; it shows approximate distances between metabolites on the SOM according to the Euclidean distance of the input vectors (Figure 3B). When we merged neighboring metabolites on the Sammon map we obtained 12 subsets of metabolites. Each subset is composed of metabolites that exhibit synchronous, time-dependent fluctuations, a "metabolic module". Metabolites in the same module were often neighbors in a traditional metabolic pathway network. Products that accumulated during the light period were arranged in subsets M1 - M8. They included the module for the reductive pentose phosphate pathway (M3), the photorespiratory pathway (M2), the latter half of the gly- 

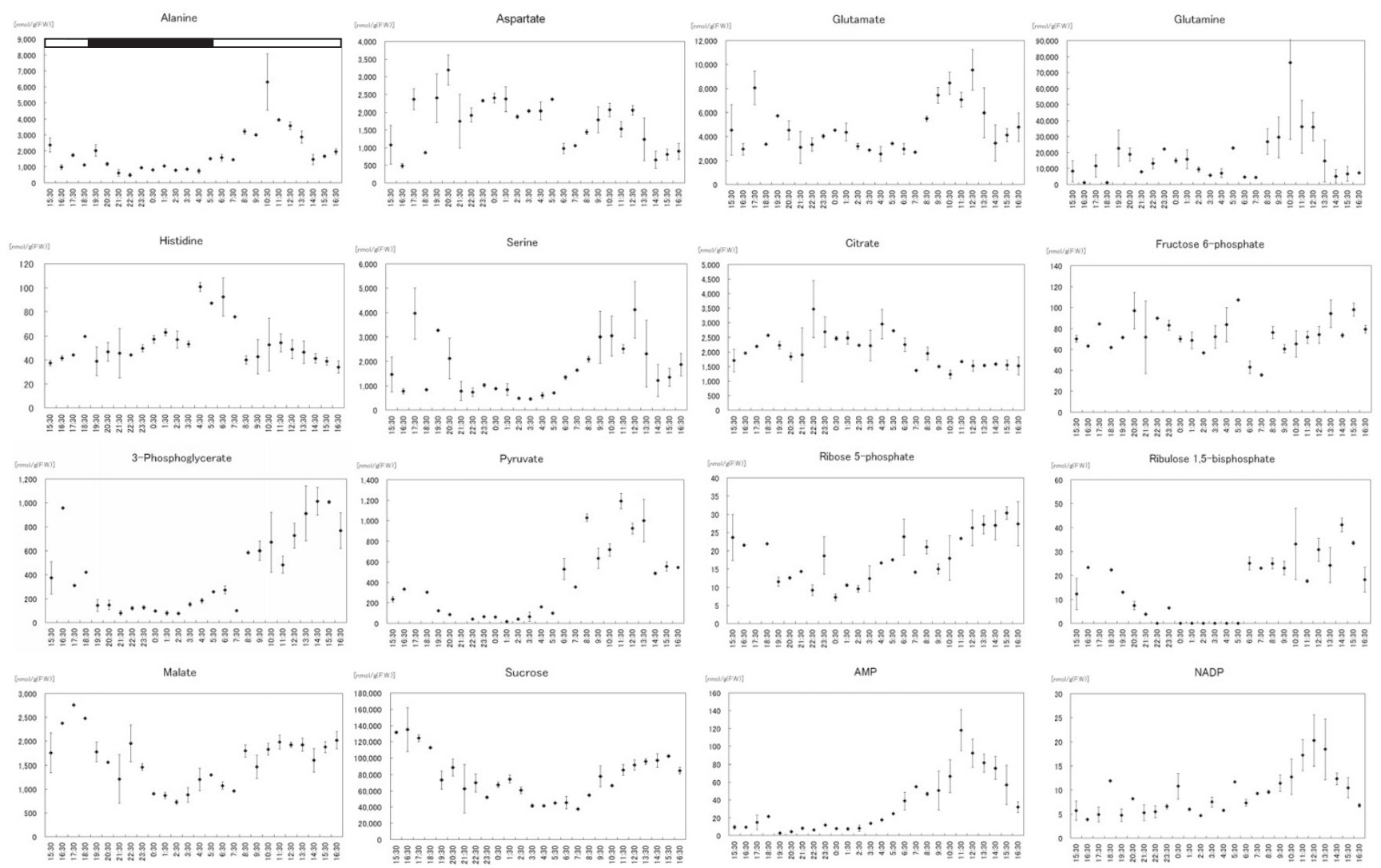

\section{Figure 2}

Metabolic time-courses in rice foliage at the third-leaf stage. Plantlets were grown under a I3-hr light - II-hr dark photocycle. We applied 3 CE-MS methods and a CE-DAD method to analyze 69 major metabolites. Dynamic changes in the metabolite levels were assessed at hourly intervals over a $24 \mathrm{~h}$ period. Averages of 2 samples $( \pm$ SEM) are shown. The top bar (shown in only $\mathrm{Ala}$ ) indicates light and dark conditions.

colytic pathway (M4), the latter half of the TCA cycle (M5), sugars (M7), and major amino acids (M1). Also included in this group were NADPH and NADH (M6), glutathione and spermidine (M8). Subsets M9 - M12 included the first half of the glycolytic pathway (M9), the first half of the TCA cycle (M10), and minor amino acids (M11); also included were the nucleoside tri- and diphosphates (M12). Thus, our SOM analysis correctly reflected

Table 3: Status of adenine nucleosides and nicotinamide coenzymes in the light and dark period

\begin{tabular}{lccccccc}
\hline & $\frac{\mathrm{ATP}}{\mathrm{AdN} * 1}$ & $\frac{\mathrm{ADP}}{\mathrm{AdN}}$ & $\frac{\mathrm{AMP}}{\mathrm{AdN}}$ & $\frac{\mathrm{NAD}}{\mathrm{NiC} * 2}$ & $\frac{\mathrm{NADH}}{\mathrm{NiC}}$ & $\frac{\mathrm{NADP}}{\mathrm{NiC}}$ & $\frac{\mathrm{NADPH}}{\mathrm{NiC}}$ \\
\hline Light*3 & 0.21 & 0.40 & 0.40 & 0.36 & 0.10 & 0.09 & 0.44 \\
Dark*4 & 0.45 & 0.43 & 0.11 & 0.55 & 0.09 & 0.05 & 0.31
\end{tabular}

*I AdN = ATP + ADP + AMP

$* 2 \mathrm{NiC}=\mathrm{NAD}+\mathrm{NADH}+\mathrm{NADP}+\mathrm{NADPH}$

$* 3$ The average of all data throughout the light period

$* 4$ The average of all data throughout the dark period the phenotypic metabolic variations that indicate functioning biochemical pathways, and therefore represents a phenotypic linkage map (PLM).

The advantages of this analysis became even more apparent upon time-resolved analysis of metabolite levels (Figure 3C), which allowed visualization of the dynamic activity of these metabolic modules (see Discussion).

\section{Discussion}

Estimation of unidentified metabolites with SOM analysis Although S17P could not be directly identified, we hypothesized that its peak could be identified in CE-MS data by combining SOM analysis with knowledge of the chemical structure. We identified a candidate peak among several peaks on selected ion electropherograms using a simple estimation method. As electrophoretic mobility is proportional to the ionic charge of the solute and inversely proportional to the size of the ionic molecule related to the hydrated ionic radius of a spherical mole- 
A

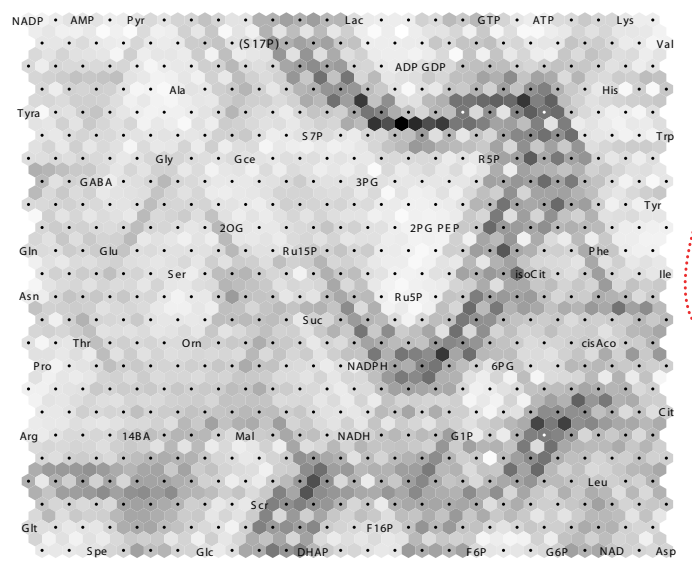

B

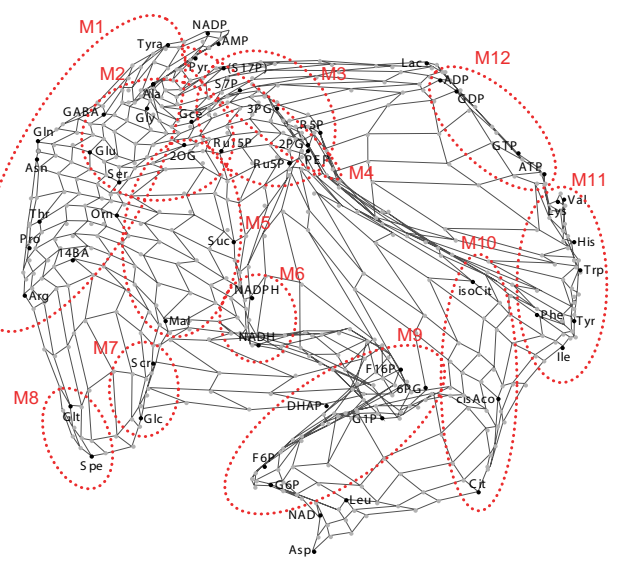

\section{C}
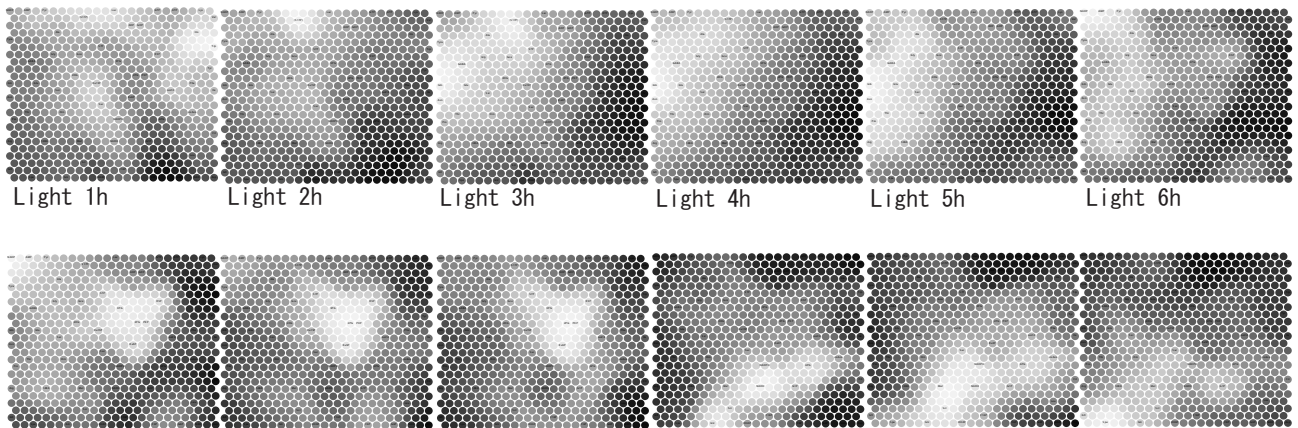

Light $7 \mathrm{~h}$

Light $8 \mathrm{~h}$

Light $9 \mathrm{~h}$

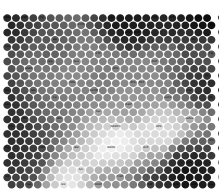

Light 5 h

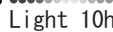

Light $11 \mathrm{~h}$

Light $12 \mathrm{~h}$
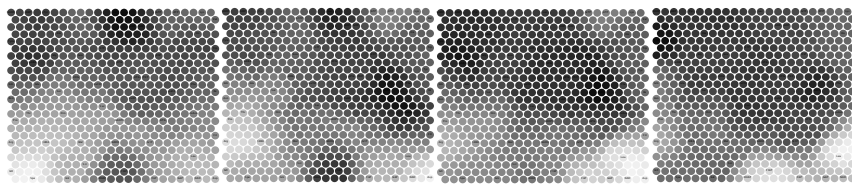

Light $13 \mathrm{~h}$

$$
\text { Dark } 1 \mathrm{~h}
$$

Dark $2 \mathrm{~h}$

Dark $3 \mathrm{~h}$

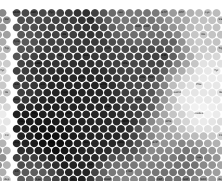

Dark 6h

Dark $8 \mathrm{~h}$

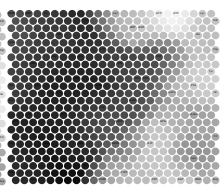

Dark $9 \mathrm{~h}$

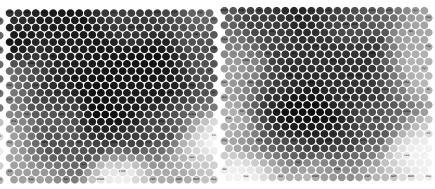

Dark $4 \mathrm{~h} \quad$ Dark $5 \mathrm{~h}$

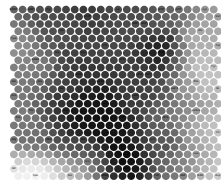

Dark $7 \mathrm{~h}$

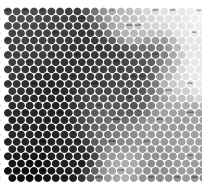

Dark $10 \mathrm{~h}$

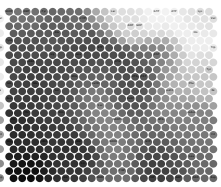

Dark $11 \mathrm{~h}$

\section{Figure 3}

Self-organizing map (SOM) Analysis. A. U-matrix. Measured metabolites $(n=56)$ were arranged in a $20 \times 20$ lattice on the basis of diurnal change similarities. Light- and dark shading indicate high and low similarity, respectively. B. Phenotypic linkage map (PLM). The linkage among metabolites based on dynamic similarity is expressed as the distance on the quadratic plane. The metabolites were assigned to 14 metabolic modules that fluctuated synchronously; most contained traditional metabolic pathway networks or similar compounds. MI, major amino acid; M2, related to photorespiratory pathway intermediates; M3, pentose phosphate pathway; M4, latter half of the glycolytic pathway; M5, latter half of the TCA cycle; M6, environmental stress response; M7, sugars; M8, NADH and NADPH; M9, first half of the glycolytic pathway; MI 0; first half of the TCA cycle; MII, minor amino acids; MI2, nucleoside tri- and diphosphates. C. Time-resolved layout. The relative levels of metabolites are shown for every time point from the start of the light period to the end of the dark period. Light and dark shading indicate high and low levels. 
cule [26], we used the cubic root of the molecular weight as a substitute parameter for the radius. Indeed, the cubic root of molecular weights of 3 metabolites of similar chemical structure, Ru5P, F6P and S7P, were linearly correlated with migration time ratios ( $r>0.999)$, when PIPES was used as an internal standard (Table 4).

The estimate for S17P was performed using linear approximation with Ru15P and F16P. The estimated migration time ratio $\left(\mathrm{MT} / \mathrm{MT}_{\mathrm{IS}}\right)$ of S17P was 0.941 (Table 4). Several peaks were observed at a mass-to-charge ratio $(\mathrm{m} / \mathrm{z})$ of 369. A peak of $M T / M_{I S}=0.909(\mathrm{~m} / \mathrm{z}=369)$ was identified within $\pm 5.0 \%$ of the predicted values.

Next, the absence of other metabolites with similar chemical structures was verified with the KEGG ligand database [27]. Note that except for S17P, metabolites were cyclic or non-anionic compounds.

Finally, we obtained the normalized time-course of the putative S17P by calculating the ratio of the peak area of putative S17P to PIPES. Integration of these data into the SOM analysis showed that this putative S17P marker was near metabolites in the reductive pentose phosphate pathway (Figure 3A) or the metabolic module M3 in PLM.

Unfortunately, the above result includes some speculation; most peaks of putative S17P were below the detection limits $(\mathrm{S} / \mathrm{N}<3)$ and the peak was not detected in the dark period. In the SOM analysis, the peak area of such undetected metabolite was calculated as zero. Nevertheless, the proposed estimation method seems to be effective in identifying unknown metabolites.

\section{Detection of metabolic bottlenecks by pair-wise correlation analysis}

In previous studies, Peason's correlation coefficients of metabolite pairs (pair-wise correlation) were applied to construct a metabolic correlation network $[5,10,28]$. A correlation coefficient is an index of co-linearity between two variables. If two metabolites, $\mathrm{A}$ and $\mathrm{B}$, are always equilibrated, i.e., $[A] /[B]=K_{e q}$ (constant), then their relationship is linear and shows a high correlation. Although real metabolic pathways are dynamic and constantly reg- ulated by their influx and/or efflux, the pathway components that are blocked by rate-limiting enzymes should exhibit approximate linearity. For example, 3PG, 2PG, and PEP in the glycolytic pathway are positioned between two rate-limiting enzymes, phosphoglycerate kinase (EC 2.7.2.3) and pyruvate kinase (PK; EC 2.7.1.40), both of which are regulated by the ATP/ADP ratio (Figure 1). The correlation coefficients among these three metabolites throughout a 24 -hr period were over 0.90 , whereas the correlation coefficient between PEP and Pyr, limited by $\mathrm{PK}$, was under 0.50. Thus, pair-wise correlation analysis is effective for the identification of metabolic modules that are regulated by rate-limiting enzymes.

We used a hierarchical clustering algorithm, Ward's method [29], to classify metabolites in the glycolytic pathway (Figure 1) on the basis of their correlation matrix that was computed using all data throughout the $24-\mathrm{hr}$ period. Indeed, a dendrogram identified the steps regulated by the ATP/ADP ratio (Figure 4A). On the other hand, it did not identify phosphofructokinase I (PFK-1; EC 2.7.1.11) as a rate-limiting enzyme. Although it is regulated by the ATP/ ADP ratio in animal cells, another enzyme, pyrophosphate fructose 6-phosphate 1-phosphotransferase (EC 2.7.1.90), seems to be active in plant cells and may be independent of the ATP/ADP ratio [30].

The same cluster analysis was also applied to the TCA cycle intermediates (Figure 1), and the dendrogram revealed the rate-limiting enzymes in the cycle again (Figure 4B): citrate synthase (CS; EC 2.3.3.1), and NADPdependent isocitrate dehydrogenase (ICDH; EC 1.1.1.42). This suggests that the classification of metabolites along enzymatic steps can help to reveal bottleneck enzymes.

\section{Time-resolved carbon/nitrogen metabolomics}

Inspection of the time-course of metabolic modules allowed us to better understand the carbon and nitrogen $(\mathrm{C} / \mathrm{N})$ assimilation/dissimilation process and their underlying function during a 24-hr period (Figure 3C).

In the first half of the light period, some accumulation emerged for carbon-fixed products: Pyr, 2OG, and photorespiratory pathway intermediates (metabolic module

Table 4: Estimated migration-time of unidentifiable metabolites based on the molecular weight of similar metabolites

\begin{tabular}{|c|c|c|c|c|}
\hline Compound & Formula & M.W. & M.W. ${ }^{1 / 3}$ & $\mathrm{MT} / \mathrm{MT}_{\mathrm{IS}}$ \\
\hline Ru5P & $\mathrm{CH}_{2}(\mathrm{OH}) \mathrm{CO}[\mathrm{CH}(\mathrm{OH})]_{2} \mathrm{CH}_{2} \mathrm{OPO}_{3} \mathrm{H}_{2}$ & 230.0192 & 6.127 & 1.029 \\
\hline F6P & $\mathrm{CH}_{2}(\mathrm{OH}) \mathrm{CO}[\mathrm{CH}(\mathrm{OH})]_{3} \mathrm{CH}_{2} \mathrm{OPO}_{3} \mathrm{H}_{2}$ & 260.0298 & 6.383 & 1.080 \\
\hline S7P & $\mathrm{CH}_{2}(\mathrm{OH}) \mathrm{CO}[\mathrm{CH}(\mathrm{OH})]_{4} \mathrm{CH}_{2} \mathrm{OPO}_{3} \mathrm{H}_{2}$ & 290.0403 & 6.619 & 1.125 \\
\hline RuI5P & $\mathrm{CH}_{2}\left(\mathrm{OPO}_{3} \mathrm{H}_{2}\right) \mathrm{CO}[\mathrm{CH}(\mathrm{OH})]_{2} \mathrm{CH}_{2} \mathrm{OPO}_{3} \mathrm{H}_{2}$ & 309.9854 & 6.768 & 0.847 \\
\hline $\mathrm{FI} 6 \mathrm{P}$ & $\mathrm{CH}_{2}\left(\mathrm{OPO}_{3} \mathrm{H}_{2}\right) \mathrm{CO}[\mathrm{CH}(\mathrm{OH})]_{3} \mathrm{CH}_{2} \mathrm{OPO}_{3} \mathrm{H}_{2}$ & 339.9960 & 6.980 & 0.895 \\
\hline SI7P & $\mathrm{CH}_{2}\left(\mathrm{OPO}_{3} \mathrm{H}_{2}\right) \mathrm{CO}[\mathrm{CH}(\mathrm{OH})]_{4} \mathrm{CH}_{2} \mathrm{OPO}_{3} \mathrm{H}_{2}$ & 370.0065 & 7.179 & $0.94 I^{*}$ \\
\hline
\end{tabular}

*Estimated value. $\mathrm{MT} / \mathrm{MT}_{\text {IS }}$ was calculated by linear approximation 


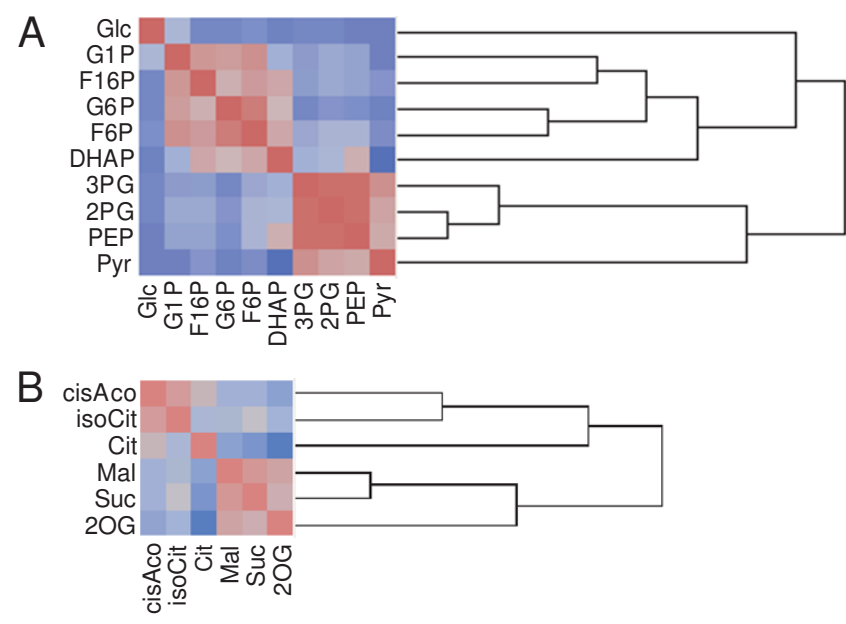

\section{Figure 4}

Hierarchical cluster analysis. A. Cluster analysis (Ward's method [26]) was applied to the correlation matrix composed of metabolic intermediates in the glycolytic pathway. The generated dendrogram was clustered into regulatory units by the ATP/ADP ratio; hexokinase (EC 2.7.I.I), phosphoglycerate kinase (EC 2.7.2.3), and pyruvate kinase (EC 2.7.I.40). B. As well as in the TCA cycle, the dendrogram was divided into two major groups at the rate-limiting steps; citrate synthase (CS; EC 2.3.3.I), and NADP-dependent isocitrate dehydrogenase (ICDH; EC I.I.I.42).

M2). This coincides with carbon fixation by activation of several light-dependent enzymes including rubisco (EC 4.1.1.39) at the start of light exposure [31], as shown by the accumulation of Ru15P, Gce and triose derivatives at the beginning of the light period (light $1-3 \mathrm{hr}$ ). The slow accumulation was partly attributable to the very slow metabolic turnover of rubisco [32]. Likewise, major amino acids and amines including Glu and Gln, the source compounds of nitrogen assimilation as amino-group acceptor/donor [33,34], also accumulated in the first half of the light period (M1). This coincides with the diurnal metabolic dynamics and the activities of key enzymes in tobacco plant [35]. For example, NR activity is known to remarkably increase immediately after the start of light exposure and decrease at midday.

On the other hand, the glycolytic pathway and the reductive pentose phosphate pathway intermediates reached their highest levels (M3, M4) at midday, and sugars peaked at the end of the light period (M7).

We can hypothesize that carbon fixed in the first half of the light period moves down the glycolytic pathway and the TCA cycle, and amino acid biosynthesis progresses using generated Glu, Pyr, and 2OG. In the latter half of the light period, the flow of fixed carbon leads to the accumulation of the intermediates in the pentose phosphate path- way and to sucrose synthesis by inhibiting the production of ammonia, Pyr, and 2OG.

From the end of the light period through the first half of the dark period, we noted an increase in sugar phosphates from the first half of the glycolytic pathway (metabolic module M9). Around midnight, the accumulation of a few organic acids in the first half of the TCA cycle (metabolic module M10) was observed, suggesting the activation of the TCA cycle.

In the latter half of the dark period, the level of minor amino acids was increased (metabolic module M11), although they are synthesized from diverse biochemical pathways. The good correlation among these minor amino acids, also reported in potato and wheat [36], is attributable to the fact that the ratio between Gln and 2OG regulate minor amino acids in bacteria and fungi through the reaction Glu +2 -oxo acid $\leftrightarrow$ amino acid + 2OG [37]. Under our experimental conditions, the Glu/ $2 \mathrm{OG}$ ratio was much higher in the dark- than in the light period (22.9 vs. 7.2 ) and the amino group can easily transferred to 2-oxo acids to produce amino acids.

\section{Adenine nucleoside and nicotinamide coenzyme status}

ATP and ADP were placed in the dark-activated group in PLM (metabolic module M12); they were accumulated at the end of the dark period, and decreased by illumination (Figure 3C). On the other hand, AMP was placed in the light-activated group peaking at midday. The reason for fluctuations of adenylate is unknown. Previous observations also do not coincide in the adenylate levels during the light- and dark period. In sugar beet leaves, all adenylate levels increased in the light period [38]. In spinach leaves and wheat leaf protoplast, ATP increased but ADP and AMP decreased under light $[39,40]$. In Crassulaceanacid metabolism (CAM) species, on the contrary, ATP decreased but ADP and AMP increased [41]. Such differences may result from different dynamics in cytosol, chloroplasts, and mitochondria [40].

We extrapolate that the lower ATP ratio during the light period was caused by an excess demand of ATP by intraand extra cellular processes for carbon fixation and nitrogen assimilation against ATP supply from photosynthesis. In theory, the amount of ATP consumption in the reductive pentose phosphate pathway and the photorespiratory pathway is more than ATP production in the photophosphorylation [42]. Beside this, nitrogen assimilation process, intracellular transport of the assimilation products, and sucrose synthesis and its translocation are also accompanied by ATP. Therefore the dark respiration makes a considerable contribution to produce ATP even in the light. However, granted that ATP supply is insufficient in the light, high metabolic turnover of adenylate 
kinase (EC 2.7.4.3) would immediately work to reproduce ATP from ADP that leads to increase of AMP. Further investigation is necessary to clarify the adenylate dynamics among cell compartments.

In our analysis, NADPH and NADH behaved similarly (metabolic module M6), whereas NADP and NAD did not. As NADPH and NADH were respectively generated by their unique reaction of reducing NADP and NAD, dependence on the intracellular oxidation-reduction state shifted the formation of oxidation and reduction. In PLM, however, NADP was placed in the light-activated- and NAD in the dark-activated group. This suggests that highly concentrated NAD in the dark is converted to NADPH via NADP in the light period. It was reported that the $\mathrm{NADPH} / \mathrm{NAD}$ ratio is the inverse of the ATP/ADP ratio in guard cell protoplast, which indicates that ATP phosphorylates NAD in the light period by NAD kinase (EC 2.7.1.23) and the generated NADP is reduced to NADPH in the course of photosynthesis [43].

The ratios of NADH to NAD and NADPH to NADP were 0.16-0.29 and 6.2-6.6. The observed difference in the tendency of oxidized- or reduced form indicates their different cellular roles. NADH is used for oxidative phosphorylation, and a low NADH/NAD ratio constrains this process. On the other hand, NADPH is used for the reductive biosynthesis of metabolites, and the high ratio of $\mathrm{NADPH} / \mathrm{NADP}$ favors the reduction of metabolites.

\section{Environmental stress response}

It is remarkable that Glt (GSH; gamma-glutamylcysteinyl glycine) and Spe exhibited similar fluctuation patterns (metabolic module M8). Both peaked at the end of the light period and again just after midnight, suggesting the existence of common regulatory factors. GSH plays a central role in the antioxidant defense by eliminating harmful peroxide during photosynthesis and oxidative phosphorylation [44]. Polyamines, including spermidine, are also effective antioxidants under various environmental stress conditions [45]. During photosynthesis, GSH is converted to oxidized dithiol (GSSH) to eliminate oxidative stress, and upon the reduction of NADPH, GSSH can be converted back to GSH by glutathione reductase (GR; EC 1.8.1.7, annotated in rice plant). Our finding that NADPH reached its highest level at a few hours before the end of the light period is consistent with the above observation (Figure 3C), although the connection remains speculative. The relative contribution of NADPH and NADH to the generation of GSH and spermidine requires further investigation.

\section{Conclusion}

We intended to analyze the rice plant metabolism and to reconstruct its phenotypic networks in an effort to explain underlying biological functions. Our CE-MS technology provided a comprehensive high-throughput system with easy sample preparation and facilitated the generation of high-resolution metabolic time-courses. Data mining with statistical techniques and SOM analysis revealed synchronous dynamics in metabolic modules downstream of $\mathrm{C}$ and $\mathrm{N}$ assimilation and dissimilation processes and stress responses. Our system was able to discriminate unidentified metabolites and identify bottleneck enzymatic steps. In a comprehensive approach such heuristics become increasingly important because with current technology, the determination of all network components is virtually impossible. For a more precise investigation of biochemical networks, expansion of target metabolites and determination of metabolite levels in each cellular compartment may be suggested. There are technical hurdles, however, in separating organelles without disturbing a wide range of metabolites inside them. Without much technical advancement, therefore, it seems difficult to repeat our time-course measurement for any single cellular compartment although there are reports for such a challenge [46]. Finally, for the analysis part, it is necessary to couple biological information with computer simulations based on large-scale time-resolved measurements of metabolites, proteins, and mRNAs.

\section{Methods \\ Plant materials}

Young seedlings of rice plants, Oryza sativa L. ssp. japonica Haenuki, at the third-leaf stage were cultured as follows. Rice seeds were germinated on filter paper soaked with Milli-Q water and kept at $30^{\circ} \mathrm{C}$ in a dark room for 2 days. After germination, the plantlets were placed on rock fiber (35 $\times 35 \times 40 \mathrm{~mm}$; Nittobo, Tokyo, Japan), and grown in a growth chamber (FLI-301N, Tokyo Rika Kikai, Tokyo, Japan) for 18 days. The temperature and light conditions were $25^{\circ} \mathrm{C}$ and $365 \mu \mathrm{E} \cdot \mathrm{m}^{-2} \mathrm{~s}^{-1}$ for $9 \mathrm{hr}$ (light), $20^{\circ} \mathrm{C}$ and 0 $\mu \mathrm{E} \cdot \mathrm{m}^{-2} \mathrm{~s}^{-1}$ for $11 \mathrm{hr}$ (dark), and $150 \mu \mathrm{E} \cdot \mathrm{m}^{-2} \mathrm{~s}^{-1}$ for $2 \mathrm{hr}$ between light and dark. The plants were watered with Kasugai water culture solution $\left(18.9 \mathrm{mg} / \mathrm{L}\left(\mathrm{NH}_{4}\right)_{2} \mathrm{SO}_{4}, 10.1\right.$ $\mathrm{mg} / \mathrm{L} \mathrm{Na}_{2} \mathrm{HPO}_{4} \cdot 12 \mathrm{H}_{2} \mathrm{O}, 4.7 \mathrm{mg} / \mathrm{L} \mathrm{KCl}, 0.79 \mathrm{mg} / \mathrm{L} \mathrm{CaCl}_{2}$, $3.0 \mathrm{mg} / \mathrm{L} \mathrm{MgCl}{ }_{2}, 0.17 \mathrm{mg} / \mathrm{L} \cdot \mathrm{FeCl}_{3} \cdot 6 \mathrm{H}_{2} \mathrm{O}$, and $\mathrm{HCl}$ to adjust the $\mathrm{pH}$ to $5.0-5.5)$ [47].

\section{Reagents}

Piperazine-1,4-bis(2-ethanesulfonic acid) (PIPES) was purchased from Dojindo (Kumamoto, Japan), methionine sulphone from Avocado Research (Heysham, Lancashire, UK). All other reagents were obtained from conventional commercial sources. Individual stock solutions, at a concentration of 10 or $100 \mathrm{mM}$, were prepared in Milli-Q water, $0.1 \mathrm{~N} \mathrm{HCl}$, or $0.1 \mathrm{~N} \mathrm{NaOH}$. The working standard mixture was prepared by diluting these stock solutions with Milli-Q water just before injection. All chemicals used were of analytical or reagent grade. Water 
was purified with a Milli-Q purification system (Millipore, Bedford, MA, USA).

\section{Sample preparation}

Leaves were harvested (fresh weight approximately 100 mg (6 seedlings)) and frozen in liquid nitrogen to stop enzymatic activity. They were mashed in a Multi-Beads Shocker (Yasuikikai, Osaka, Japan) at $2000 \mathrm{rpm}$ for $10 \mathrm{sec}$ and $0.5 \mathrm{~mL}$ of ice-cooled methanol, including $400 \mu \mathrm{M}$ PIPES and methionine sulphone as an internal standard, was added to dissolve phospholipid membranes and inactive enzymes. Then $0.5 \mathrm{~mL}$ ice-cold Milli-Q water was added and the sample was ultrafiltered through a $5-\mathrm{kDa}$ cut-off filter at $9058 \mathrm{~g}$ for $10 \mathrm{~min}$ to remove proteins, phospholipids, chlorophyll, and other high-molecularweight impurities. The filtrate was analyzed by CE-MS and CE-DAD methods. To obtain sufficient sensitivity for the analysis of nucleotides, coenzymes, and sugars, the filtrate was concentrated 5 -fold by lyophilization [17].

\section{Instruments}

All CE-MS experiments were performed by Agilent CE capillary electrophoresis. We used a 1100 series MSD mass spectrometer, a 1100 series isocratic HPLC pump, a G1603A CE-MS adapter kit, and a G1607A CE-ESI-MS sprayer kit (Agilent Technologies). CE-DAD experiments were performed by Agilent CE capillary electrophoresis with a built-in diode-array detector. G2201AA Agilent ChemStation software for CE was used for system control, data acquisition and analysis, and MSD data evaluation.

\section{Analytical conditions}

The compounds were analyzed in four groups using three CE-MS methods and one CE-DAD method.

a) Cationic metabolites (amino acids and amines) were analyzed with a fused-silica capillary $(50 \mu \mathrm{m}$ i.d. $\times 100 \mathrm{~cm}$ total length), with $1 \mathrm{M}$ formic acid as the electrolyte. The sample was injected at an injection pressure of $5.0 \mathrm{kPa}$ for $3 \mathrm{sec}$ (approximately $3 \mathrm{~nL}$ ). The applied voltage was set at $30 \mathrm{kV}$. The capillary temperature was set to $20^{\circ} \mathrm{C}$, and the sample tray was cooled to below $5^{\circ} \mathrm{C}$. The sheath liquid ( $5 \mathrm{mM}$ ammonium acetate in $50 \%$ [v/v] methanol-water) was delivered at $10 \mu \mathrm{L} / \mathrm{min}$. ESI-MS was conducted in positive ion mode; the capillary voltage was set at $4000 \mathrm{~V}$. A flow rate of heated dry nitrogen gas (heater temperature $300^{\circ} \mathrm{C}$ ) was maintained at $10 \mathrm{~L} / \mathrm{min}[12]$.

b) Anionic metabolites (organic acids and sugar phosphates) were analyzed with a cationic polymer-coated SMILE(+) capillary (Nakalai Tesque, Kyoto, Japan). The electrolyte for CE separation was a $50 \mathrm{mM}$ ammonium acetate solution ( $\mathrm{pH} 8.5)$. The sample was injected at an injection pressure of $5.0 \mathrm{kPa}$ for $30 \mathrm{sec}$ (approximately 30 $\mathrm{nL})$. The applied voltage was set at $-30 \mathrm{kV}$, and the capil- lary temperature was set to $30^{\circ} \mathrm{C}$. ESI-MS was conducted in negative ion mode; the capillary voltage was set at 3500 $\mathrm{V}$. Other conditions were as in the cationic metabolite analysis [13].

c) Nucleotides and coenzymes were analyzed with an uncharged polymer-coated gas chromatograph capillary, polydimethylsiloxane (DB-1) (Agilent Technologies). The electrolyte for CE separation was $50 \mathrm{mM}$ ammonium acetate solution ( $\mathrm{pH} 7.5)$. The applied voltage was set at -30 $\mathrm{kV}$ and a pressure of $5.0 \mathrm{kPa}$ was added to the inlet capillary during the run. Other conditions were as in the anion analysis [14].

d) Sugars were analyzed with a fused-silica capillary (50 $\mu \mathrm{m}$ i.d. $\times 112.5 \mathrm{~cm}$ total length, $104 \mathrm{~cm}$ effective length). Basic anion buffer for CE (Agilent Technologies) was the electrolyte. The sample was injected at a pressure of 5.0 $\mathrm{kPa}$ for $10 \mathrm{sec}$ (approximately $10 \mathrm{~nL}$ ). The applied voltage was set at $-25 \mathrm{kV}$; the capillary temperature, regulated with a thermostat, was $25^{\circ} \mathrm{C}$. Sugars were detected by indirect UV detection using a diode-array detector. The signal wavelength was set at $350 \mathrm{~nm}$ with a reference at $230 \mathrm{~nm}$ [48].

\section{Self-organizing map (SOM) analysis}

A free software package, SOM -PAK [49], was used to compute both the SOM and the Sammon map. Before SOM analysis, the observed time-course data for 58 metabolites (including an estimate of S17P) were smoothed by averaging the adjacent data points using a sliding window of width 3, to reduce high-frequency noise presumably originating from individual differences in plant seedlings, rapid oscillations in metabolism, or measurement errors. The missing data points were extrapolated by linear approximation between prior and subsequent data values. Among the 57 metabolites evaluated at 26 time points, only 30 data points could be extrapolated due to the detection limit or contamination of other unidentifiable peaks. The SOM is a map from the input $n$-dimensional data space (input layer) to a two-dimensional array of nodes (output layer). The vectors in the output layer are the parametric reference vector $\boldsymbol{m}_{i}$, which has $n$ elements. An input data vector, $x$, is compared with $\boldsymbol{m}_{i}$, and the bestmatch vector, which is the smallest Euclidean distance $\mid x$ $\boldsymbol{m}_{\boldsymbol{i}} \mid$, is mapped onto this location. During learning, nodes that are topographically close in the array up to a certain distance activate each other to learn from the same input vector, and the reference vectors are corrected so that they become close to the input vector. Thus,

$$
\boldsymbol{m}_{i}(\mathrm{t}+1)=\boldsymbol{m}_{i}(\mathrm{t})+h_{c i}(\mathrm{t})\left[x(\mathrm{t})-\boldsymbol{m}_{i}(\mathrm{t})\right],
$$

where $\mathrm{t}$ is an integer, the discrete-time coordinate, and $h_{c i}(t)$ is the neighborhood kernel, a function defined over 
the lattice points. The neighborhood size, $N_{c^{\prime}}$ around node $c$ is a function of time, and $h_{c i}$ is defined as

$$
\begin{array}{ll}
h_{c i}=\alpha(\mathrm{t}) & \left(i \in N_{c}\right) \\
h_{c i}=0 & \left(i \notin N_{c}\right),
\end{array}
$$

where $\alpha(\mathrm{t})$ is a monotonic decreasing function of time ( 0 $<\alpha(\mathrm{t})<1)$ called the "learning rate". The learning rate function was defined as

$$
\alpha(t)=\alpha(0)(1.0-t / T)
$$

where $\alpha(0)$ is the initial learning rate and $T$ the running length (number of steps) in training. In this study, 58 metabolic time-courses were formatted and classified in a 24 $\times 24$ hexagonal lattice. The applied SOM parameters were: initial radius of the training area $=12$, initial learning rate $=0.025$, running length $=65000$.

\section{Metabolic pair-wise correlation}

Significance levels for Pearson correlation coefficient $r$ were computed depending on the number of metabolite pairs $n$ found throughout the light and dark period, respectively, by calculating t-scores given by $t=r(n-2)^{0.5} /$ $(1-r)^{0.5}$. The critical t-score was set to correspond to the commonly used p-value of 0.05 in two-sided tests.

\section{Hierarchical clustering}

Among several algorithms for clustering analysis, we chose Ward's method [29] in JMP software (ver. 6.0.0; SAS Institute Inc. Cary, NC). Starting from trivial clusters each containing one object only, Ward's method iteratively merges two clusters that will result in the smallest increase in the sum of the square of their differences (i.e., variance). At each step, all possible mergers of two clusters are tried and their variance is computed. The difference between clusters is calculated by the equation:

$$
d(a, b)=\frac{n_{a} n_{b}}{n_{a}+n_{b}}\left(x_{a}-x_{b}\right)^{2}
$$

\section{Authors' contributions}

SS conceived this study, performed the biochemical- and the computational experiments, and wrote the manuscript. MA provided intellectual help for the computational analysis and together wrote the manuscript. TN advised the experimental design. TS and MT supervised the research. All authors read and approved the final manuscript.

\section{Acknowledgements}

This work was supported by a grant for the Development of Rice Genome Simulators from MAFF, Japan, and by the Ministry of Education, Culture, Sports, Science and Technology, and a Grant-in-Aid for the 2 Ist Century Center of Excellence (COE) Program entitled "Understanding and Control of Life's Function via Systems Biology (Keio University)". This work was also supported, in part, by Grant-in-Aid for Scientific Research on Priority Areas "Systems Genomics" from the Ministry of Education, Culture, Sports, Science and Technology of Japan.

\section{References}

I. Fridman E, Pichersky E: Metabolomics, genomics, proteomics, and the identification of enzymes and their substrates and products. Curr Opin Plant Biol 2005, 8(3):242-248.

2. Raamsdonk LM, Teusink B, Broadhurst D, Zhang N, Hayes A, Walsh MC, Berden JA, Brindle KM, Kell DB, Rowland J], Westerhoff HV, Dam K, Oliver SG: A functional genomics strategy that uses metabolome data to reveal the phenotype of silent mutations. Nat Biotechnol 200I, 19(I):45-50.

3. Allen J, Davey HM, Broadhurst D, Heald JK, Rowland JJ, Oliver SG, Kell DB: High-throughput classification of yeast mutants for functional genomics using metabolic footprinting. Nat Biotechnol 2003, 21 (6):692-696.

4. Morgenthal K, Wienkoop S, Scholz M, Selbig J, Weckwerth W: Correlative GC-TOF-MS-based metabolite profiling and LC-MSbased protein profiling reveal time-related systematic regulation of metabolite-protein networks and improve pattern recognition for multiple biomarker selection. Metabolomics 2005, I(2): 109-12I.

5. Weckwerth W, Loureiro ME, Wenzel K, Fiehn O: Differential metabolic networks unravel the effects of silent plant phenotypes. Proc Natl Acad Sci USA 2004, I 0 I (20):7809-78I4.

6. Hirai MY, Klein M, Fujikawa Y, Yano Y, Goodenowe DB, Yamazaki Y, Kanaya S, Nakamura Y, Kitayama M, Suzuki H, Sakurai N, Shibata D, Tokuhisa J, Reichelt M, Gershenzon J, Papenbrock J, Saito K: Elucidation of gene-to-gene and metabolite-to-gene networks in arabidopsis by integration of metabolomics and transcriptomics. I Biol Chem 2005, 280(27):25590-25595.

7. Kohonen T: Self-Organizing Maps Springer-Verlag, Heidelberg, Germany; 1995.

8. Mounet F, Lemaire-Chamley M, Maucourt M, Cabasson C, Giraude JL, Deborde C, Lessire R, Gallusci P, Bertrand A, Gaudillère M, Rothan C, Rolin D, Moing A: Quantitative metabolic profiles of tomato flesh and seeds during fruit development: Complementary analysis with ANN and PCA. Metabolomics 2007, 3(3):273-288.

9. Panagiotou G, Kouskoumvekaki I, Jónsdóttir , Olsson L: Monitoring novel metabolic pathways using metabolomics and machine learning: Induction of the phosphoketolase pathway in Aspergillus nidulans cultivations. Metabolomics 2007, 3(4):503-516.

10. Fiehn O: Metabolic networks of Cucurbita maxima phloem. Phytochemistry 2003, 62(6):875-886.

II. Yeung KY, Medvedovic M, Bumgarner RE: From co-expression to co-regulation: How many microarray experiments do we need? Genome Biol 2004, 5(7):R48.

12. Soga T, Heiger DN: Amino acid analysis by capillary electrophoresis electrospray ionization mass spectrometry. Anal Chem 2000, 72: $1236-124 \mid$.

13. Soga T, Ueno Y, Naraoka H, Ohashi Y, Tomita M, Nishioka T: Simultaneous determination of anionic intermediates for Bacillus subtilis metabolic pathway by capillary electrophoresis electrospray ionization mass spectrometry. Anal Chem 2002, 74:2233-2239.

14. Soga T, Ueno Y, Naraoka H, Matsuda K, Tomita M, Nishioka T: Pressure-assisted capillary electrophoresis electrospray ionization mass spectrometry for analysis of multivalent anions. Anal Chem 2002, 74:6224-6229.

15. Soga T, Ohashi Y, Ueno Y, Naraoka H, Tomita M, Nishioka T: Quantitative metabolome analysis using capillary electrophoresis mass spectrometry. J Proteome Res 2003, 2:488-494.

16. Ishii N, Nakahigashi K, Baba T, Robert M, Soga T, Kanai A, Hirasawa T, Naba M, Hirai K, Hoque A, Ho PY, Kakazu Y, Sugawara K, Igarashi S, Harada S, Masuda T, Sugiyama N, Togashi T, Hasegawa M, Takai Y, Yugi K, Arakawa K, Iwata N, Toya Y, Nakayama Y, Nishioka T, Shimizu K, Mori H, Tomita M: Multiple high-throughput analyses monitor the response of E. coli to perturbations. Science 2007, 316(5824):593-7.

17. Sato S, Soga T, Nishioka T, Tomita M: Simultaneous determination of the main metabolites in rice leaves using capillary 
electrophoresis mass spectrometry and capillary electrophoresis diode array detection. The Plant J 2004, 40:15I-163.

18. Sammon JW Jr: A nonlinear mapping for data structure analysis. IEEE Transactions Computers 1969, C-1 8(5):40I-409.

19. KEGG pathway database [http://www.genome.ad.jp/kegg/path way.html]

20. Swiss-Prot database [http://au.expasy.org/sprot/]

21. Rice Annotation Project Data Base [http://rapdb.dna.affrc.go.jp/

22. Stafford HA, Magaldi A, Vennesland B: The enzymatic reduction of hydroxypyruvic acid to $D^{-g l y c e r i c}$ acid in higher plants. Biol Chem 1954, 207:621-629.

23. Rippert $P$, Matringe M: Purification and kinetic analysis of the two recombinant arogenate dehydrogenase isoforms of Arabidopsis thaliana. Eur J Biochem 2002, 269:4753-476I.

24. Boldt R, Edner C, Kolukisaoglu Ü, Hagemann M, Weckwerth W, Wienkoop S, Morgenthal K, Bauwe H: -Glycerate 3-kinase, the last unknown enzyme in the photorespiratory cycle in Arabidopsis, belongs to a novel kinase family. The Plant Cell 2005, 17:2413-2420.

25. Duncan K, Edwards RM, Coggins JR: The pentafunctional arom enzyme of Saccharomyces cervisiae is a monofunctional domains. Biochem J 1987, 246:375-386.

26. Kuhr WG: Separation of small organic molecules. In Capillary Electrophoresis: Theory and Practice Edited by: Camilleri P. CRC Press LLC, Boca Raton, FL, USA; 1997:9I-I33.

27. KEGG ligand database [http://www.genome.ad.jp/kegg/lig and.html]

28. Steuer R, Kurths J, Fiehn O, Weckwerth W: Observing and interpreting correlations in metabolic networks. Bioinformatics 2003, 19(8): 1019-1026.

29. Ward $\mathrm{JH}$ : Hierarchical grouping to optimize an objective function. J Am Stat Assoc 1963, 58:236-245.

30. Ashihara H, Sato F: Pyrophosphate: Fructose-6-phosphate Iphosphotransferase and biosynthetic capacity during differentiation of hypocotyls of Vigna seedlings. Biochim Biophys Acto 1993, II 56:123-127.

3I. Farr TJ, Huppe HC, Turpin DH: Coordination of chloroplastic metabolism in $\mathbf{N}$-limited chlamydomonas reinhardtii by redox modulation (I. The activation of phosphoribulokinase and glucose-6-phosphate dehydrogenase is relative to the photosynthetic supply of electrons). Plant Physiol 1994 105:1037-1042.

32. Woodrow IE, Berry J: Enzymatic regulation of photosynthetic $\mathrm{CO}_{2}$ fixation in $\mathrm{C}_{3}$ plants. Ann Rev Plant Physiol Plant Molec Biol 1988, 39:533-594.

33. Lea PJ, Ireland RJ: Nitrogen metabolism in higher plants. In Plant Amino Acids, Biochemistry and Biotechnology Edited by: Singh BK. New York: Marcel Dekker, Inc; 1999: I-47.

34. Ireland RJ, Lea PJ: The enzymes of glutamine, glutamate, asparagines, and aspartate metabolism. In Plant Amino Acids, Biochemistry and Biotechnology Edited by: Singh BK. New York: Marcel Dekker, Inc; 1999:49-109.

35. Scheible WR, Krapp A, Stitt M: Reciprocal diurnal changes of phosphoenol pyruvate carboxylase expression and cytosolic pyruvate kinase, citrate synthase and NADP-isocitrate dehydrogenase expression regulate organic acid metabolism during nitrate assimilation in tobacco leaves. Plant Cell and Environ 2000, 23: I155-1167.

36. Noctor G, Novitskaya L, Lea PJ, Foyer CH: Co-ordination of leaf minor amino acid contents in crop species: Significance and interpretation. J Exp Bot 2002, 53(370):939-945.

37. Ferrario-Méry S, Suzuki A, Kunz C, Valadier MH, Roux $Y$, Hirel B, Foyer $\mathrm{CH}$ : Modulation of amino acid metabolism in transformed tobacco plants deficient in Fd-GOGAT. Plant and Soil 2000, $221: 67-79$.

38. Rao IM, Arulanantham AR, Terry N: Diurnal changes in adenylates and nicotinamide nucleotides in sugar beet leaves. Photosynthesis Res 1990, 23:205-2/2.

39. Bonzon M, Hug M, Wagner E, Greppin H: Adenine nucleotides and energy charge evolution during the induction of flowering in spinach leaves. Planta 198I, 152:189-194.

40. Stitt M, Lilley RM, Heldt HW: Adenine nucleotide levels in the cytosol, chloroplasts, and mitochondria of wheat leaf protplasts. Plant Physiol 1982, 70:97|-977.

4I. Chen LS, Nose A: Day-Night changes of energy-rich compounds in crassulacean acid metabolism (CAM) species utilizing hexose and starch. Ann Bot 2004, 94:449-455.

42. Leegood RC: Photosynthesis in $\mathrm{C}_{3}$ plants: The Benson-Calvin cycle and photorespiration. In Plant Biochemistry and Molecular Biology 2nd edition. Edited by: Lea PJ, Leegood RC. John Wiley \& Sons Ltd; 1999:29-50.

43. Hampp R, Schnabl H: Adenine and pyridine nucleotide status of isolated Vicia guard cell protoplasts during $\mathrm{K}+$-induced swelling. Plant and Cell Physiol I984, 25(7): I233-1239.

44. May MJ, Vernoux T, Leaver C, Montagu MV, Inze D: Glutathione homeostasis in plants: Implications for environmental sensing and plant development. J Exp Bot 1998, 49(32I):649-667.

45. Lovaas E: Antioxidant and metal-chelating effects of polyamines. In Advances in Pharmacology. Antioxidants in Disease Mechanisms and Therapy 38 Edited by: Sies H. New York: Academic Press; 1996:119-149.

46. Farré EM, Tiessen A, Roessner U, Geigenberger P, Trethewey RN, Willmitzer L: Analysis of the compartmentation of glycolytic intermediates, nucleotides, sugars, organic acids, amino acids, and sugar alcohols in potato tubers using a nonaqueous fractionation method. Plant Physiol 200I, I 27:685-700.

47. Kasugai S: Studies of water culture. Jpn / Soil Sci Plant Nutr 1939, 13:669-822. (in Japanese)

48. Soga T, Ross GA: Simultaneous determination of inorganic anions, organic acids, amino acids and carbohydrate by capillary electrophoresis. J Chromatogr A 1999, 837:23I-239.

49. SOM-PAK [http://www.cis.hut.fi/research/som lvq pak]

Publish with Biomed Central and every scientist can read your work free of charge

"BioMed Central will be the most significant development for disseminating the results of biomedical research in our lifetime. "

Sir Paul Nurse, Cancer Research UK

Your research papers will be:

- available free of charge to the entire biomedical community

- peer reviewed and published immediately upon acceptance

- cited in PubMed and archived on PubMed Central

- yours - you keep the copyright
BioMedcentral 